\title{
Coordination Control of Discrete-Event Systems
}

\author{
Jan Komenda \\ Institute of Mathematics, Czech Academy of Sciences, \\ Brno Branch, Zizkova 22, 61662 Brno, Czech Republic \\ komenda@ipm. cz
}

\author{
Jan H. van Schuppen \\ CWI, P.O. Box 94079 , \\ 1090 GB Amsterdam, The Netherlands \\ J.H.van.Schuppen@cwi.nI
}

\begin{abstract}
The concept of a coordinator is proposed for control of modular discrete-event systems. The coordinator makes all subsystems conditionally independent generators as defined in the paper. The coordinator receives part of the partial observations of the subsystems and its task is to satisfy the global part of the specification and of the nonblockingness. The complete supervisor then consists of the coordinator, its supervisor, and the local supervisors for the subsystems. An example of control of a distributed discrete-event system shows that a coordinator is necessary for achieving safety and nonblockingness.
\end{abstract}

\section{INTRODUCTION}

The purpose of this paper is to present the coordination control approach for control of decentralized and modular discrete-event systems. Because of the complexity of control problems and because of the way large engineering systems are constructed, there is an increasing interest in control of decentralized and modular systems.

Our previous results in modular control of DES rely on necessary and sufficient conditions for local control synthesis to equal global control synthesis for both local and global specifications and for both complete and partial observations. Interestingly, we have shown recently in [7] that the structural conditions used for global specifications are equivalent to those used for local specification. However if these conditions are not satisfied, either the system must be modified to meet them or a hierarchical approach (as the one that was proposed in [11]) should be adopted such that the abstracted system meets these structural conditions. Moreover blocking issues have not been considered in our earlier approach.

Therefore we have developed an approach based on coordination to handle the blocking issues. Coordination of discrete-event systems was discussed earlier by Kai C. Wong and W. Murray Wonham in [14]. A similar approach to ours has also been proposed in [4], where the authors have shown that if all projections from local alphabets that abstract away events which are not included in the coordinator sets are observers of the local systems then there always exists a coordinator which makes the composed system nonblocking. The main difference is that our method provides both sufficient and necessary conditions on the coordinator to make the composed system nonblocking.

The research was supported by Grant Agency of Acad. of Sci. of Czech Republic and Acad. of Sci. of Czech Republic, Inst. Research Plan No. AV0Z10190503.
Coordination control is based on the concept of conditional independence of $\sigma$-algebras which is used in probability theory. The coordinator generator makes two or more generators conditionally independent if the joint action of two or more generators is always accompanied by a transition of the coordinator. Conditional independence can also be defined for automata languages. A coordinator always exists so the search is for the smallest possible coordinator. The novelty of the paper is in the concept of a coordinator for safety and for nonblockingness based on the concept of conditional independence. The approach is inspired by the work of K.C. Wong, W.M. Wonham, and coworkers on modular control, by research of the authors of this paper on modular control, and by publications of K. Schmidt and T. Moor on a hierarchical approach to control, see [12].

A description of the contents follows. The next section presents a problem formulation and motivation of the problem. In Section III the basic concepts of the paper: conditional independence of languages and of generators is introduced. In Section IV these concepts are studied and related to each other. Section V shows how nonblocking can be achieved by composing the modular plant with a suitable coordinator. In Section VI safety is studied. The control synthesis of the coordinator with supervisors is discussed in Section VI. The proofs are included in the appendix.

\section{PROBLEM FORMULATION}

The motivation for coordination control is decentralized and modular control of discrete-event systems (DES). Consider a modular DES. It is well known, see the lecture notes of W.M. Wonham, [15], that a supervisor for the modular system for which a controller has been synthesized using modular control, may be blocking. Example 5.5 in Section V establishes that there does not exist a set of local supervisors which is nonblocking unless the observations of both models are sent to a global coordinator who can then prevent blocking by disabling particular events. Thus the nonblocking property of modular control cannot always be achieved modularly and nonblockingness requires a coordinator at the global level. See for modular control of DES [8] and [2].

The main problem for both decentralized control and modular control is the construction of a coordinator. A coordinator always exists, a supervisor for the global plant will be a coordinator. The difficult part of the problem is to 
construct a minimal coordinator which is least restrictive in regard to the local subsystems.

\section{Terminology and Notation}

The terminology of DES is more or less according to the lecture notes of W.M. Wonham [15] and the book [1] but the notation of this paper differs slightly. A (deterministic) generator

$$
G=\left(Q, E, f, q_{0}, Q_{m}\right),
$$

is a mathematical structure with state set $Q$, an event set $E$, a partial transition function $f: Q \times E \rightarrow Q$, an initial state $q_{0} \in Q$, and a subset of marked states $Q_{m} \subseteq Q$. A transition is also denoted as $q \stackrel{e}{\longmapsto} q^{+}=f(q, e)$. If a transition is defined then this is denoted by $f(q, e)$ ! Extend the transition function $f$ to $f: Q \times E^{*} \rightarrow Q$ by induction. Define respectively the language and the marked language of the generator as,

$$
\begin{aligned}
L(G) & =\left\{s \in E^{*} \mid f\left(q_{0}, s\right) !\right\}, \\
L_{m}(G) & =\left\{s \in L(G) \mid f\left(q_{0}, s\right) \in Q_{m}\right\} .
\end{aligned}
$$

A controlled generator is a structure $\left(G, E_{c}, \Gamma_{c}\right)$, where $G$ is a generator, $E_{c} \subseteq E$ is the subset of controllable events, $E_{u c}=E \backslash E_{c}$ is the subset of uncontrollable events, and $\Gamma_{c}=$ $\left\{\gamma \subseteq E \mid E_{u c} \subseteq \gamma\right\}$, is called the set of control patterns. A supervisor for the controlled generator is map $g: L(G) \rightarrow$ $\Gamma_{c}$. The closed-loop system associated with a controlled generator and a supervisor as denoted above is defined as the smallest language $L(S / G) \subseteq E^{*}$ and the marked language $L_{m}(S / G) \subseteq L(S / G)$ which satisfy respectively,

$$
\begin{aligned}
& \varepsilon \in L(S / G), \\
& \text { if } s \in L(S / G), \text { se } \in L(G) \text { and if } e \in g(s) \\
& \text { then } s e \in L(S / G) .
\end{aligned}
$$$$
L_{m}(S / G)=L(S / G) \cap L_{m}(G) .
$$

Recall that the natural projection $P: E^{*} \rightarrow E_{o}^{*}$ is a morphism of monoids such that $P(\varepsilon)=\varepsilon$ and $P$ erases the events that are not in $E_{o} \subseteq E$. A supervisor with partial observations is a map $g: P(L(G)) \rightarrow \Gamma_{c}$.

It is important to distinguish for a generator between an event set and its associated reachable event set. Note that there may exist events of the event set which do not appear in any transition. Moreover, even if an event is used for a transition then that transition may not be reachable. This applies in particular to the case of the synchronous product of two generators.

Definition 2.1: Consider a generator $G$ denoted as above. Define the subset of reachable events, denoted by $E_{r}(G) \subseteq E$, if for any event $e \in E_{r}(G)$ there exists a string $s \in E^{*}$ containing the event $e$ for which the function $f\left(q_{0}, s\right)$ is defined. Similarly, define for any language $L \subseteq E^{*}$ the subset of reachable events $E_{r}(L) \subseteq E$ of the language as the subset of events which occur in the strings of the language.

Note the abuse of notation in $E_{r}(G)$ and $E_{r}(L)$. The complexity of computing the event set $E_{r}(G)$ is $O\left(n(G) \times m_{E}(G)\right)$ where $n(G)$ denotes the number of states and $m_{E}(G)$ denotes the number of events of the generator $G$.
Definition 2.2: Consider two event sets $E_{1}$ and $E_{2}$ and two languages $L_{1} \subseteq E_{1}^{*}$ and $L_{2} \subseteq E_{2}^{*}$. The synchronous product of the languages $L_{1}$ and $L_{2}$ is defined as

$$
L_{1} \| L_{2}=P_{1}^{-1}\left(L_{1}\right) \cap P_{2}^{-1}\left(L_{2}\right) .
$$

where $P_{i}:\left(E_{1} \cup E_{2}\right)^{*} \rightarrow E_{i}^{*}$ for $i=1,2$. Their synchronous product is called the shuffle product if

$$
\begin{aligned}
\emptyset= & E_{r, s h}=E_{r}\left(L_{1} \| L_{2}\right) \cap E_{1} \cap E_{2}, \\
& \text { and then one writes }
\end{aligned}
$$$$
\operatorname{shuffle}\left(L_{1}, L_{2}\right)=L_{1} \| L_{2}=P_{1}^{-1}\left(L_{1}\right) \cap P_{2}^{-1}\left(L_{2}\right) .
$$

The subset of reachable shared events $E_{r, s h}$ can be empty while $E_{1} \cap E_{2}$ is not as a simple example shows. The corresponding synchronous product of generators [15] is known to satisfy

$$
\begin{aligned}
L\left(G_{1} \| G_{2}\right) & =L\left(G_{1}\right) \| L\left(G_{2}\right), \\
L_{m}\left(G_{1} \| G_{2}\right) & =L_{m}\left(G_{1}\right) \| L_{m}\left(G_{2}\right) .
\end{aligned}
$$

A distributed discrete-event system is a modular or a concurrent system with the global plant formed by the synchronous product of local subsystems.

Definition 2.3: A modular discrete-event system with two modules is a structure $\left(G_{1}, G_{2}, E_{1, c}, \Gamma_{1, c}, E_{2, c}, \Gamma_{2, c}\right)$ consisting of two modules in the form of controlled generators. The associated global system is their synchronous product $G_{1} \| G_{2}$. Denote the natural projections by

$$
P_{1}:\left(E_{1} \cup E_{2}\right)^{*} \rightarrow E_{1}^{*}, P_{2}:\left(E_{1} \cup E_{2}\right)^{*} \rightarrow E_{2}^{*} .
$$

Throughout the paper the special case of two modules is considered in order to simplify the exposition. A coordinator of a modular system will be illustrated in Example 5.5.

\section{CONCEPTS}

In this section the concept of conditionally independent generators and related notions are defined.

Conditional independence of $\sigma$-algebras is a concept of probability theory which has been used to put the concept of state of a stochastic system on a fundamental basis, see [9] and the references quoted there. A corresponding notion is useful in automata theory as well. This section presents the concepts, coordination control theory with these concepts is presented in the following sections.

Denote $E=E_{1} \cup E_{2}$ and $E_{k} \subseteq E$ a coordinator alphabet. The following natural projections are needed: $P_{1 \cup k}: E^{*} \rightarrow$ $\left(E_{1} \cup E_{k}\right)^{*}, P_{1}^{1 \cup k}:\left(E_{1} \cup E_{k}\right)^{*} \rightarrow E_{1}^{*}$, and the corresponding inverse projection $\left(P_{1}^{1 \cup k}\right)^{-1}: \operatorname{Pwr}\left(E_{1}^{*}\right) \rightarrow \operatorname{Pwr}\left(E_{1} \cup E_{c}\right)^{*}$. Similarly, $P_{2 \cup k}: E^{*} \rightarrow\left(E_{2} \cup E_{k}\right)^{*}, P_{2}^{2 \cup k}:\left(E_{2} \cup E_{k}\right)^{*} \rightarrow E_{2}^{*}$, and $\left(P_{2}^{2 \cup k}\right)^{-1}: \operatorname{Pwr}\left(E_{2}^{*}\right) \rightarrow \operatorname{Pwr}\left(E_{2} \cup E_{k}\right)^{*}$ are defined. Symmetrically, let $P_{k}^{i \cup k}:\left(E_{i} \cup E_{k}\right)^{*} \rightarrow E_{k}^{*}, i=1,2$. Also, let $P_{i \backslash k}: E^{*} \rightarrow$ $\left(E_{i} \backslash E_{k}\right)^{*}, i=1,2$. The notation $P_{i \cap k}^{i}: E_{i}^{*} \rightarrow\left(E_{i} \cap E_{k}\right)^{*}, i=1,2$ is now self-explanatory.

Definition 3.1: Consider three generators,

$$
\begin{aligned}
& G_{k}=\left(Q_{k}, E_{k}, f_{k}, q_{k, 0}, Q_{k, m}\right), \\
& G_{1}=\left(Q_{1}, E_{1}, f_{1}, q_{1,0}, Q_{1, m}\right), \\
& G_{2}=\left(Q_{2}, E_{2}, f_{2}, q_{2,0}, Q_{2, m}\right) .
\end{aligned}
$$


Call $G_{1}, G_{2}$ conditionally independent generators given $G_{k}$ if for any reachable state in the synchronous product $\left(q_{k}, q_{1}, q_{2}\right) \in G_{k}\left\|G_{1}\right\| G_{2}$, there does not exist a transition of the form,

$$
\left(q_{k}, q_{1}, q_{2}\right) \mapsto\left(q_{k}, q_{1}^{+}, q_{2}^{+}\right),
$$

and $a \notin E_{k}$

Note that conditional independence means that there is no simultaneous move in both $G_{1}$ and $G_{2}$ without the coordinator being also involved. The concept is easily extended to the case of three or more generators. The corresponding concept in terms of languages follows.

Definition 3.2: Consider event sets $E_{1}, E_{2}, E_{k}$ and languages $L_{1} \subseteq E_{1}^{*}, L_{2} \subseteq E_{2}^{*}$, and $L_{k} \subseteq E_{k}^{*}$. The languages $L_{1}, L_{2}$ are said to be conditionally independent given $L_{k}$ if $E_{r}\left(L_{1} \| L_{2}\right) \cap E_{1} \cap E_{2} \subseteq E_{k}$.

Notation. $\left(L_{1}, L_{2} \mid L_{k}\right) \in \mathrm{CIL}$ denotes that the languages $L_{1}, L_{2}$ are conditionally independent given $L_{k}$.

Other related concepts are defined below.

Definition 3.3: Consider the events sets $E_{1}, E_{2}$ and $E_{k}$ and the languages $L_{1} \subseteq E_{1}^{*}, L_{2} \subseteq E_{2}^{*}, L_{k} \subseteq E_{k}^{*}$, and $K \subseteq E^{*}$. Assume that $E_{r}\left(L_{1} \| L_{2}\right) \cap E_{1} \cap E_{2} \subseteq E_{k} \subseteq E_{1} \cup E_{2}=E$. Define the conditions:

(a) The triple of languages $\left(L_{1}, L_{2}, L_{k}\right)$ is called conditionally shuffle closed if

$$
\begin{aligned}
& t \in L_{1}\left\|L_{2}\right\| L_{k}, \text { with decomposition, } \\
t= & s_{1} t_{c 1} \ldots s_{k} t_{c k} s_{k+1}, \\
& s_{1}, \ldots s_{k+1} \in\left[\left(E_{1} \backslash E_{k}\right) \cup\left(E_{2} \backslash E_{k}\right)\right]^{*}, \\
& t_{c, 1}, \ldots, t_{c, k} \in E_{k}^{*} \\
\operatorname{PS}\left(s_{i}\right) \in \quad & \operatorname{shuffle}\left(P_{1 \backslash k}\left(s_{i}\right), P_{2 \backslash k}\left(s_{i}\right)\right), \forall i=1, \ldots, k+1, \\
\bar{t} \in & \operatorname{PS}\left(s_{1}\right) t_{c 1} \ldots \operatorname{PS}\left(s_{k}\right) t_{c, k} \operatorname{PS}\left(s_{k+1}\right) \\
\Rightarrow & \bar{t} \in L_{1}\left\|L_{2}\right\| L_{k} .
\end{aligned}
$$

(b) The triple of languages $\left(L_{1}, L_{2}, L_{k}\right)$ is called conditionally projection-closed if for all $t, \bar{t} \in E^{*}, t \in L_{1}\left\|L_{2}\right\| L_{k}$, $P_{k}(t)=P_{k}(\bar{t}), P_{1 \cup k}(t)=P_{1 \cup k}(\bar{t})$, and $P_{2 \cup k}(t)=P_{2 \cup k}(\bar{t})$ imply that $\bar{t} \in L_{1}\left\|L_{2}\right\| L_{k}$.

(c) The language $K$ is called conditionally decomposable with respect to the event sets $\left(E_{1}, E_{2}, E_{k}\right)$ if

$$
K=P_{1 \cup k}(K)\left\|P_{2 \cup k}(K)\right\| P_{k}(K) .
$$

The term conditionally decomposable is used, because the projections $P_{i \cup k}: E^{*} \rightarrow E_{i \cup k}^{*}$ are involved. It should be clear that conditional decomposability is weaker than decomposability with respect to $P_{1}: E^{*} \rightarrow E_{1}^{*}, P_{2}: E^{*} \rightarrow E_{2}^{*}$, and $P_{k}: E^{*} \rightarrow E_{k}^{*}$ as defined in the literature on decentralized control. This is because $E_{i} \subseteq E_{i} \cup E_{k}$ implies that $P_{i}^{-1} P_{i}(K) \subseteq$ $P_{i \cup k}^{-1} P_{i \cup k}(K)$. Also note that in case $E_{k}=E=E_{1} \cup E_{2}$ conditional decomposability is trivially satisfied.

\section{EQUIVALENT CONDITIONS FOR CONDITIONAL INDEPENDENCE}

The concepts of conditional indepence and conditional decomposability are closely related. First of all we notice from the very definitions that the following Proposition holds.
Proposition 4.1: Consider the generators of Definition 3.1. The following statements are equivalent:

(a) $G_{1}$ and $G_{2}$ are conditionally independent given $G_{k}$.

(b) The languages $\left(L\left(G_{1}\right), L\left(G_{2}\right) \mid L\left(G_{k}\right)\right) \in$ CIL are conditionally independent.

(c) $E_{r}\left(G_{1} \| G_{2}\right) \cap E_{1} \cap E_{2} \subseteq E_{k}$.

Theorem 4.2: Consider the events sets $E_{1}, E_{2}$ and $E_{k}$ and the languages $L_{1} \subseteq E_{1}^{*}, L_{2} \subseteq E_{2}^{*}$, and $L_{k} \subseteq E_{k}^{*}$.

(a) If the languages $L_{1}, L_{2}$ are conditionally independent given $L_{k}$ then the triple $\left(L_{1}, L_{2}, L_{k}\right)$ is conditionally shuffle closed.

(b) Assume that the languages $L_{1}, L_{2}$ are conditionally independent given $L_{k}$. Then the following statements are equivalent:

(b.1) The triple $\left(L_{1}, L_{2}, L_{k}\right)$ is conditionally shuffle closed.

(b.2) The triple $\left(L_{1}, L_{2}, L_{k}\right)$ is conditionally projection closed.

(b.3) The language $L_{1}\left\|L_{2}\right\| L_{k}$ is conditionally decomposable.

The technical results below will be needed in the remaining sections.

Lemma 4.3: [3, Proposition 4.3] Let $E_{1} \cap E_{2} \subseteq E_{k}$ and $L_{i} \subseteq E_{i}^{*}, i=1,2$. Then $P_{k}\left(L_{1} \| L_{2}\right)=P_{1 \cap k}^{1}\left(L_{1}\right) \| P_{2 \cap k}^{2}\left(L_{2}\right)$.

Let us note that the condition $E_{1} \cap E_{2} \subseteq E_{k}$ in the above lemma can be weakened to our condition $E_{r}\left(G_{1} \| G_{2}\right) \cap E_{1} \cap$ $E_{2} \subseteq E_{k}$ : some of the events from $E_{1} \cap E_{2}$ might actually not be reachable in $G_{1} \| G_{2}$ and these need not be included in $E_{k}$ for the lemma to hold true.

Lemma 4.4: Let $L_{i} \subseteq E_{i}^{*}, i=1,2, k$ and $E_{r}\left(G_{1} \| G_{2}\right) \cap E_{1} \cap$ $E_{2} \subseteq E_{r}\left(G_{k}\right)$. Then

(1) $P_{k}\left(L_{1}\left\|L_{2}\right\| L_{k}\right) \subseteq L_{k}$

(2) $P_{1 \cup k}\left(L_{1}\left\|L_{2}\right\| L_{k}\right) \subseteq L_{1} \| L_{k}$

(3) $P_{2 \cup k}\left(L_{1}\left\|L_{2}\right\| L_{k}\right) \subseteq L_{2} \| L_{k}$

\section{COORDINATOR FOR NONBLOCKINGNESS}

In this section the nonblockingness of the complete system is studied. The complete system consists of the coordinator in parallel composition with the two modules. In the following definition the equivalent condition of nonblockingness is stated. Consider again two generators and their coordinator,

$$
\begin{aligned}
& G_{1}=\left(Q_{1}, E_{1}, f_{1}, q_{1,0}, Q_{1, m}\right), \\
& G_{2}=\left(Q_{2}, E_{2}, f_{2}, q_{2,0}, Q_{2, m}\right), \\
& G_{k}=\left(Q_{k}, E_{k}, f_{k}, q_{k, 0}, Q_{k, m}\right) .
\end{aligned}
$$

Definition 5.1: We say that $G=G_{1} \| G_{2}$ is conditional nonblocking given the coordinator automaton $G_{k}$ if

(1) $\forall s \in \bar{L}_{m}\left(G_{1}\right)\left\|\bar{L}_{m}\left(G_{2}\right)\right\| \bar{L}_{m}\left(G_{k}\right) \quad \exists t_{k} \in E_{k}^{*}: P_{k}(s) t_{k} \in$ $L_{m}\left(G_{k}\right)$ and

(2) (i) conditional nonblockingness of $G_{1} \| G_{k}$ holds, i.e. $\exists v \in\left(E_{1} \cup E_{k}\right)^{*}$ such that $P_{1}(s) P_{1}^{1 \cup k}(v) \in L_{m}\left(G_{1}\right)$ and $P_{k}^{1 \cup k}(v)=t_{k}$ and

(ii) conditional nonblockingness of $G_{2} \| G_{k}$ holds, i.e. $\exists w \in\left(E_{2} \cup E_{k}\right)^{*}$ such that $P_{2}(s) P_{2}^{2 \cup k}(w) \in L_{m}\left(G_{2}\right)$ and $P_{k}^{2 \cup k}(w)=t_{k}$. 
The conditional nonblockingness represents a good compromise between existence of local prolongations to local marked states (i.e. local nonblocking) and the existence of global prolongations to marked states (i.e. global nonblocking). It is well known that local nonblocking is not enough to ensure the global nonblocking. Therefore a coordinator layer is added to the modular system such that the coordinator together with the first subsystem as well as coordinator with the second subsystem are nonblocking in the sense of the definition 5.1. The following theorem then states that the conditional nonblockingness defined above is not only sufficient, but also necessary for the nonblockingness of the composed system $G_{k}\left\|G_{1}\right\| G_{2}$.

Theorem 5.2: Consider the setting of Definition 5.1. Assume that the coordinator $G_{k}$ makes $G_{1}$ and $G_{2}$ conditionally independent, i.e. $G_{1}, G_{2}$ are conditionally independent generators given $G_{k}$ in the sense of Definition 3.1. The composed system $G_{1}\left\|G_{2}\right\| G_{k}$ is nonblocking if and only if $G$ is conditional nonblocking given $G_{k}$.

In the last theorem the nonblocking coordinator, which makes the resulting system nonblocking, is characterized using the conditions (2) (i) and (ii) of Def. 5.1. The first question is whether this characterization (in terms of strings) can be verified. Next a procedure will be given (with immediate automata interpretation), which checks whether conditions (1) and (2): (i) and (ii) above are satisfied. The key steps will be computations of coreachable sets and projected generators (automata) which can be computed using a standard subset construction. Let us recall that inverse projections of automata are obtained for free, just by adding selfloops of events not observable with respect to the corresponding projection.

We show that the candidate sets for the strings $t_{k}, v$, and $w$ obeying the properties (i) and (ii) can be found in the following way. For any $s \in \bar{L}_{m}\left(G_{1}\right)\left\|\bar{L}_{m}\left(G_{2}\right)\right\| \bar{L}_{m}\left(G_{k}\right)$ we have $P_{i}(s) \in \bar{L}_{m}\left(G_{i}\right), i=1,2$ and $P_{k}(s) \in \bar{L}_{m}\left(G_{k}\right)$. We look for extensions of $P_{i}(s) i=1,2, k$ within $L_{m}\left(G_{i}\right), i=1,2, k$, i.e.

$$
\left.S_{i}(s):=\left\{t_{i} \in E_{i}^{*}: P_{i}(s) t_{i} \in L_{m}\left(G_{i}\right)\right)\right\} .
$$

These languages can be easily computed using coreachable sets in their corresponding automata: CoReachset $\left(Q_{i, m}, E_{i}^{*}\right)$ and intersecting them with the quotient language of $G_{i}$ by $P_{i}(s), i=1,2, k$ represented by states in the automata $G_{i}$ after the strings $P_{i}(s) \in E_{i}^{*}, i=1,2, k$ have been generated. Hence, $S_{i}(s), i=1,2, k$ are fairly easily computable.

Now, the candidates for the strings $t_{k}, v$, and $w$ of Definition 5.1 can be found in terms of $S_{i}(s), i=1,2$ and suitable projections and inverse projections. Hence, we consider the intersection $P_{1 \cup k}^{-1}\left(S_{1}\right) \cap P_{2 \cup k}^{-1}\left(S_{2}\right)$, which can be implemented by the standard automata composition. The following simple fact is needed.

Property 5.3: For languages $L_{i} \subseteq E^{*}, i=1,2$ and natural projection $P_{k}: E^{*} \rightarrow E_{o}^{*}$ with $E_{o} \subseteq E$ we have $s \in P\left(L_{1}\right) \cap$ $L_{2} \neq \emptyset \Rightarrow P^{-1}(s) \subseteq L_{1} \cap P^{-1}\left(L_{2}\right) \neq \emptyset$.

Proof: Let $s \in P\left(L_{1}\right) \cap L_{2} \neq \emptyset$. Then $s \in L_{2}$ and there exists $t \in L_{1}$ such that $s=P(t)$. Hence, $t=P^{-1}(s) \subseteq P^{-1}\left(L_{2}\right)$, i.e. $t \in L_{1} \cap P^{-1}\left(L_{2}\right) \neq \emptyset$.
We obtain a computable criterion for checking the necessary and sufficient condition of Theorem 5.2.

Proposition 5.4: $G_{1} \| G_{2}$ is conditional nonblocking given the coordinator automaton $G_{k}$ if and only if

$$
\begin{aligned}
& \forall s \in \bar{L}_{m}\left(G_{1}\right)\left\|\bar{L}_{m}\left(G_{2}\right)\right\| \bar{L}_{m}\left(G_{k}\right), \\
& {\left[P_{k}^{1 \cup k}\left(P_{1}^{1 \cup k}\right)^{-1}\left(S_{1}(s)\right) \cap S_{k}(s)\right] \cap} \\
& \cap\left[P_{k}^{2 \cup k}\left(P_{2}^{2 \cup k}\right)^{-1}\left(S_{2}(s)\right) \cap S_{k}(s)\right] \neq \emptyset .
\end{aligned}
$$

It is important that the criterion of the Proposition 5.4 is checkable, because $S_{i}(s), i=1,2, k$ can be constructed using coreachable sets and all projections, inverse projections and intersections can be computed as has been pointed out above.

Let us now illustrate the above decribed procedure and action of the coordinator on the example 5.5.

Example 5.5: Let us consider the following local plant languages.

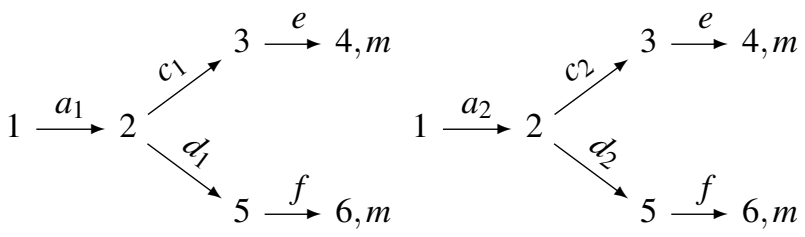

$$
\begin{aligned}
& E_{1}=\left\{a_{1}, c_{1}, d_{1}, e, f\right\}, E_{2}=\left\{a 2, c_{2}, d_{2}, e, f\right\}, \\
& E_{k}=\left\{e, f, c_{1}, c_{2}, d_{1}, d_{2}\right\} \supseteq E_{1} \cap E_{2}=\{e, f\}, \\
& G_{1} \| G_{2}, \text { blocking. }
\end{aligned}
$$

We assume that the subset of marked states are $Q_{1 m}=\{4,6\}$ and $Q_{2 m}=\{4,6\}$, which are denoted by small $m$. It is easily seen that the synchronous product of these local plants involve blocking. 


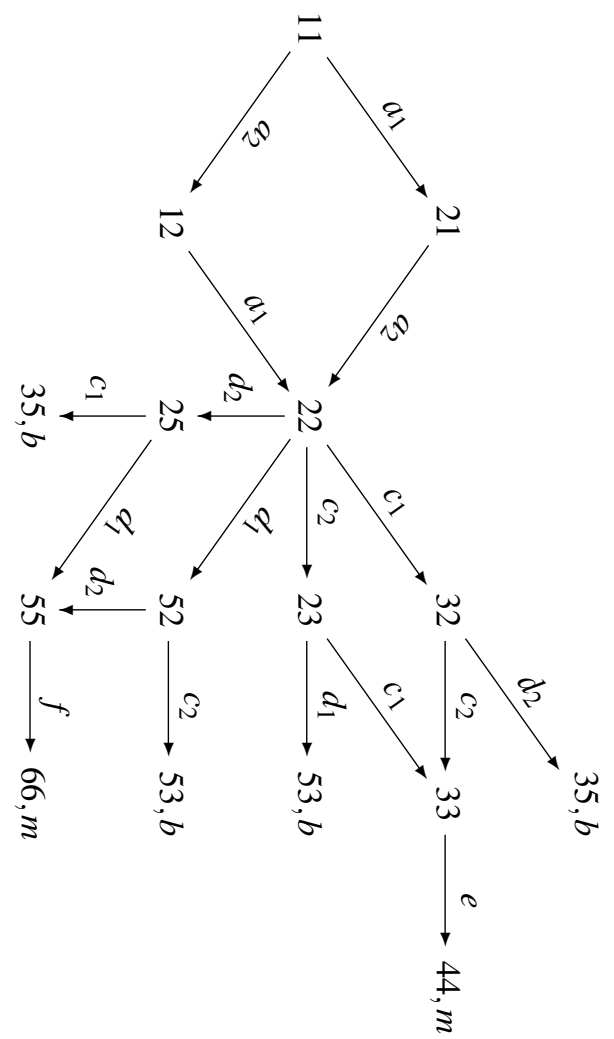

It can be easily seen that not all private events have to be included into the coordinator (high level) event set $E_{k}$. Indeed, $a_{1}$ and $a_{2}$ need not be included in $E_{k}$. The choice $E_{k}=E \backslash\left\{a_{1}, a_{2}\right\}$ is optimal for the composed system. Indeed, the following choice of $G_{k}$ is optimal such that the composed system $G_{1}\left\|G_{2}\right\| G_{k}$ is nonblocking.

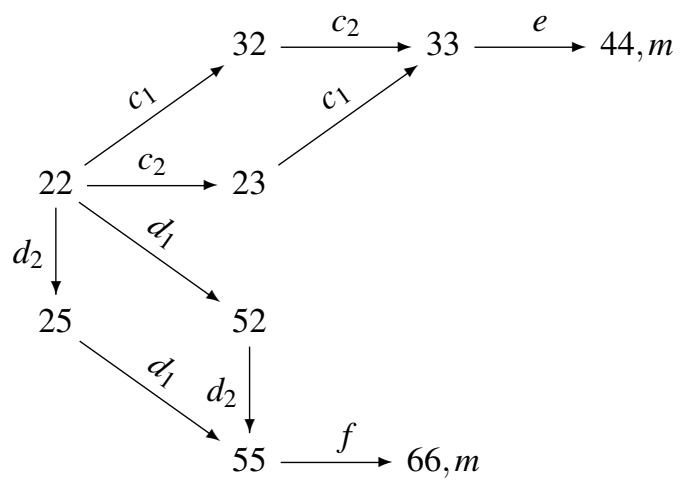

Let us also illustrate on this example the presentation of Proposition 5.4. Consider the string $s=a_{1} a_{2} c_{1}$. Then $P_{1}(s)=a_{1} c_{1}$ and $P_{k}(s)=c_{1}$. Furthermore, $S_{k}(s)=\left\{t \in E_{k}^{*} \mid c_{1} t \in L_{m}\left(G_{k}\right)\right\}=\left\{c_{2} e\right\}, S_{1}(s)=\{t \in$ $\left.E_{1}^{*} \mid a_{1} c_{1} t \in L_{m}\left(G_{1}\right)\right\}=\{e\}$. Hence, $\left(P_{1}^{1 \cup k}\right)^{-1}\left(S_{1}(s)\right)=$ $\left(c_{2}^{*} d_{2}^{*}\right)^{*} e\left(c_{2}^{*} d_{2}^{*}\right)^{*} \quad$ and $\quad P_{k}^{1 \cup k}\left(P_{1}^{1 \cup k}\right)^{-1}\left(S_{1}(s)\right)=\left\{c_{2} e\right\}$. Similarly, $P_{2}(s)=a_{2}$, i.e. $S_{2}(s)=\left\{t \in E_{2}^{*} \mid a_{2} t \in\right.$ $\left.L_{m}\left(G_{2}\right)\right\}=\left\{c_{2} e, d_{2} f\right\} \quad$ and $P_{k}^{2 \cup k}\left(P_{2}^{2 \cup k}\right)^{-1}\left(S_{2}(s)\right)=$ $\left(c_{1}^{*} d_{1}^{*}\right)^{*} c_{2}\left(c_{1}^{*} d_{1}^{*}\right)^{*} e\left(c_{1}^{*} d_{1}^{*}\right)^{*} \cup\left(c_{1}^{*} d_{1}^{*}\right)^{*} d_{2}\left(c_{1}^{*} d_{1}^{*}\right)^{*} f\left(c_{1}^{*} d_{1}^{*}\right)^{*}$. Therefore, $P_{k}^{2 \cup k}\left(P_{2}^{2 \cup k}\right)^{-1}\left(S_{2}(s)\right) \cap S_{k}(s)=\left\{c_{2}, e\right\}$. It is sufficient to take $t_{k}=c_{2} e$ and the corresponding strings $v$ and $w$ satisfying (i) and (ii) of Definition 5.1, which are $v=c_{2} e$ and $w=c_{2} e$. The role of the coordinator is to allow after the string $s=a_{1} a_{2} c_{1}$, i.e. in the corresponding state of $G_{2}$, only the string $c_{2}$, which can be then extended to the marked string $a_{1} a_{2} c_{1} c_{2} e$, while disable string $d_{2}$, which leads to blocking.

The conclusion is then $P_{k}^{1 \cup k}\left(P_{1}^{1 \cup k}\right)^{-1}\left(S_{1}(s)\right) \cap$ $P_{k}^{2 \cup k}\left(P_{2}^{2 \cup k}\right)^{-1}\left(S_{2}(s)\right) \cap S_{k}(s) \neq \emptyset$. This can be shown for any other strings $s \in \bar{L}_{m}\left(G_{1}\right)\left\|\bar{L}_{m}\left(G_{2}\right)\right\| \bar{L}_{m}\left(G_{k}\right)$, which means by Proposition 5.4 and Theorem 5.2 that $G=G_{1} \| G_{2}$ is conditional nonblocking given $G_{k}$.

Yet another important problem is the construction of the coordinator, i.e. how the coordinator can actually be synthesized in an automated manner. First of all, such a coordinator clearly exists.

Proposition 5.6: Consider a modular DES as in Definition 2.3. There exists a generator $G_{k}$, denoted as in Definition 3.1, such that $G_{1}, G_{2}$ are conditionally independent generators given $G_{k}$. In fact, $G_{k}=\operatorname{trim}\left(G_{1} \| G_{2}\right)$, i.e. the part of the global system that is both reachable and coreachable, certainly works as a coordinator for nonblockingness.

The results of [3] are helpful. The question is whether/how our condition is related to the observer property from [13]. In particular, whether there is a sufficient condition for modular nonblockingness in terms of the observer properties of suitable projections. Let us recall first the definition of an observer.

Definition 5.7: For a language $L \subseteq E^{*}$, the natural projection $P: L \rightarrow P(L) \subseteq E_{o}^{*}$ is said to be an $L$-observer if for any $t \in P(L)$ and any $s \in \bar{L}$ such that $P(s) \leq t$ there exists $u \in E^{*}$ with the properties $s u \in L$ and $t=P(s u)$.

This means that for any abstracted extension within the abstracted system's language there must exist a compatible extension within the language of the "original" system, whence the idea to adapt the observer property used in hierarchical systems for use in modular and distributed systems. If $P_{i \cap c}^{i}$ is a $L_{i}$-observer for $i=1,2$ then we know from [3, Proposition 4.10] that there always exist a coordinator. Namely, $G_{k}:=P_{i \cap c}^{i}\left(L_{1}\right) \| P_{i \cap c}^{i}\left(L_{2}\right)$ is a good choice of coordinator for nonblockingness of the composed system $G_{1}\left\|G_{2}\right\| G_{k}$.

Since the above condition is not necessary (unlike our characterizations (i) and (ii)), it follows that our conditions are weaker. On the other hand if $P_{i \cap k}^{i}$ are $L_{i}$-observers for $i=1,2$ then our conditions as necessary and sufficient for nonblocking must be satisfied.

\section{VERIFICATION FOR SAFETY}

The purpose of this subsection is to present a condition for safety of concurrent discrete-event systems. We are interested in safety of modular systems composed with the coordinator. It will be assumed that a closed-loop system is specified both for the coordinator and for the remaining parts of the subsystems after the coordinator is imposed. Control synthesis of the coordinator and the supervisors will be discussed in section VII.

An equivalent condition for verification of safety is conditional safety defined below as safety of the coordinator 
and safety of each local subsystem when combined with the coordinator.

Problem 6.1: Consider two generators $G_{1}, G_{2}$, and a coordinator $G_{k}$, which makes $G_{1}$ and $G_{2}$ conditionally independent. Consider a specification language $K \subseteq E^{*}$. Assume that the language $K$ is conditionally decomposable with respect to the reachable event sets $\left(E_{k}, E_{1}, E_{2}\right)$. Determine sufficient or equivalent conditions such that

$L_{m}\left(G_{1}\right)\left\|L_{m}\left(G_{2}\right)\right\| L_{m}\left(G_{k}\right) \subseteq K$.

We have first the following result.

Proposition 6.2: Let $K \subseteq E^{*}$ be conditionally decomposable with respect to the reachable event sets $\left(E_{k}, E_{1}, E_{2}\right)$ and let there exist a coordinator $G_{k}$ over $E_{k}^{*}$ such that

(i) $L_{m}\left(G_{k}\right) \subseteq P_{k}(K)$

(ii) $L_{m}\left(G_{1}\right) \| L_{m}\left(G_{k}\right) \subseteq P_{1 \cup k}(K)$

(iii) $L_{m}\left(G_{2}\right) \| L_{m}\left(G_{k}\right) \subseteq P_{2 \cup k}(K)$

Then $L_{m}\left(G_{1}\right)\left\|L_{m}\left(G_{2}\right)\right\| L_{m}\left(G_{k}\right) \subseteq K$.

Now we give weaker (string based) necessary and sufficient conditions for safety similar to conditions used for nonblockingness.

Definition 6.3: Consider the setting of Problem 6.1. The system and the specification language $K \subseteq E^{*}$ are said to be conditionally safe with respect to the reachable event sets $\left(E_{k}, E_{1}, E_{2}\right)$ if

(1) $L_{m}\left(G_{k}\right) \subseteq P_{k}(K)$

(2) $\forall t_{k} \in L_{m}\left(G_{k}\right)$ : $v \in L_{m}\left(G_{1}\right)\left\|L_{m}\left(G_{k}\right), P_{k}^{1 \cup k}(v)=t_{k}, w \in L_{m}\left(G_{2}\right)\right\| L_{m}\left(G_{k}\right)$, $P_{k}^{2 \cup k}(w)=t_{k} \Rightarrow v \in P_{1 \cup k}(K)$ and $w \in P_{2 \cup k}(K)$.

Note that conditional safety is weaker than (i)-(iii) of Proposition 6.2. Indeed, while (i) and (1) are the same, (2) of Definition 6.3 is weaker than (ii) and (iii) of Proposition 6.2. This is because the assumptions of the implication are stronger than in the implications corresponding to (ii) and (iii) of Proposition 6.2, i.e. $v \in L_{m}\left(G_{1}\right) \| L_{m}\left(G_{k}\right) \Rightarrow v \in P_{1 \cup k}(K)$ and idem for (iii) separately. Note that (2) is equivalent to the following inclusions:

2(i) $L_{m}\left(G_{1}\right) \| L_{m}\left(G_{k}\right) \cap\left(P_{k}^{1 \cup k}\right)^{-1} P_{k}^{2 \cup k}\left(L_{m}\left(G_{2}\right) \| L_{m}\left(G_{k}\right)\right) \subseteq P_{1 \cup k}(K)$ 2(ii) $\quad L_{m}\left(G_{2}\right) \| L_{m}\left(G_{k}\right) \cap\left(P_{k}^{2 \cup k}\right)^{-1} P_{k}^{1 \cup k}\left(L_{m}\left(G_{1}\right) \| L_{m}\left(G_{k}\right)\right) \subseteq$ $P_{2 \cup k}(K)$.

The main result of this section is now formulated below.

Theorem 6.4: Consider Problem 6.1. If the system and the specification language be conditionally safe with respect to the reachable event sets $\left(E_{k}, E_{1}, E_{2}\right) \quad$ then $L_{m}\left(G_{1}\right)\left\|L_{m}\left(G_{2}\right)\right\| L_{m}\left(G_{k}\right) \subseteq K$. Conversely, if $L_{m}\left(G_{1}\right)\left\|L_{m}\left(G_{2}\right)\right\| L_{m}\left(G_{k}\right) \subseteq K \quad$ and $L_{m}\left(G_{k}\right) \subseteq P_{k}\left(L_{m}\left(G_{1}\right) \| L_{m}\left(G_{2}\right)\right)$, then the specification language are conditionally safe with respect to the reachable event sets $\left(E_{k}, E_{1}, E_{2}\right)$.

Let us remark that $L_{m}\left(G_{k}\right) \subseteq P_{k}\left(L_{m}\left(G_{1}\right) \| L_{m}\left(G_{2}\right)\right)$ is often satisfied in coordination control, because coordinators for safety as well as coordinators for nonblockingness typically do not add additional behavior to the composed systems, i.e. $L_{m}\left(G_{k}\right)$ is included in the projected behavior.

\section{CONTROL SYNTHESIS}

In this section the overall control synthesis is presented. Using the coordination scheme, first a supervisor for coordinator is synthesized that takes care of the part $P_{k}(K)$ of the specification $K$. Then $S_{i}, i=1,2$ are synthesized such that the remaining part of the specification, i.e. $P_{i \cup k}(K)$ are met by the new plant languages $G_{i} \|\left(S_{k} / G_{k}\right)$. Let $E_{u} \subseteq E$ be the set of uncontrollable events and $E_{i, u}=E_{u} \cap E_{i}, i=1,2, k$ the corresponding sets of local uncontrollable events.

Problem 7.1: Consider generators $G_{1}, G_{2}, G_{k}$ and a specification language $K \subseteq\left(E_{1} \cup E_{2} \cup E_{k}\right)^{*}$. Assume that the coordinator $G_{k}$ makes the two generators $G_{1}, G_{2}$ conditionally independent and that the language $\bar{K}$ is conditionally decomposable.

Determine supervisors $S_{1}, S_{2}, S_{k}$ for the respective generators such that the closed-loop system with $S_{k} / G_{k}$ as coordinator for $S_{1} / G_{1}$ and $S_{2} / G_{2}$ is such that

$L\left(S_{1} /\left[G_{1} \|\left(S_{k} / G_{k}\right)\right]\right)\left\|L\left(S_{2} /\left[G_{2} \|\left(S_{k} / G_{k}\right)\right]\right)\right\| L\left(S_{k} / G_{k}\right)=\bar{K}$

Definition 7.2: Consider the setting of Problem 7.1 Call the specification language $K \subset E^{*}$ conditionally controllable for generators $\left(G_{1}, G_{2}, G_{k}\right)$ and for the event subsets $\left(E_{1, u}, E_{2, u}, E_{k, u}\right)$ if

1) The language $P_{k}(K) \subseteq E_{k}^{*}$ is controllable with respect to $G_{k}$ and $E_{k, u}$; equivalently,

$$
\overline{P_{k}(K)} E_{k, u} \cap L\left(G_{k}\right) \subseteq \overline{P_{k}(K)} .
$$

Then there then exists a nonblocking supervisor $S_{k}$ for $G_{k}$ such that $L\left(S_{k} / G_{k}\right)=\overline{P_{k}(K)}$. The supervisor $S_{k}$ is used in the remaining part of the definition.

2) The language $P_{1 \cup k}(K) \subseteq\left(E_{1} \cup E_{k}\right)^{*}$ is controllable with respect to $L\left(G_{1} \|\left(S_{k} / G_{k}\right)\right)$ and $E_{1+k, u}=E_{u} \cap\left(E_{1} \cup E_{k}\right)$; equivalently,

$$
\overline{P_{1 \cup k}(K)} E_{1+k, u} \cap L\left(G_{1} \|\left(S_{k} / G_{k}\right)\right) \subseteq \overline{P_{1 \cup k}(K)}
$$

3) The language $P_{2 \cup k}(K) \subseteq\left(E_{2} \cup E_{k}\right)^{*}$ is controllable with respect to $L\left(G_{2} \|\left(S_{k} / G_{k}\right)\right)$ and $E_{2+k, u}=E_{u} \cap\left(E_{2} \cup E_{k}\right)$; equivalently,

$$
\overline{P_{2 \cup k}(K)} E_{2+k, u} \cap L\left(G_{2} \|\left(S_{k} / G_{k}\right)\right) \subseteq \overline{P_{2 \cup k}(K)}
$$

The conditions of Definition 7.2 can be checked by algorithms as is directly clear from the computational complexity of controllability in the case of only one subsystem. The computational complexity of checking conditional controllability is much less than that of the global system, $L\left(G_{1}\right)\left\|L\left(G_{2}\right)\right\| L\left(G_{k}\right)$. This is because instead of checking the controllability with global specification and system we check it only on the corresponding projections to $E_{k} \cup E_{1}$ and $E_{k} \cup E_{2}$. The projections are smaller when they satisfy the observer property.

Theorem 7.3: Consider Problem 7.1 of control for safety. There exists a set of supervisors $\left(S_{k}, S_{1}, S_{2}\right)$ such that

$$
\left.L\left(S_{1} /\left[G_{1} \|\left(S_{k} / G_{C}\right)\right]\right)\left\|L\left(S_{2} /\left[G_{2} \|\left(S_{k} / G_{C}\right)\right]\right)\right\| L\left(S_{k} / G_{k}\right)\right)=\bar{K},
$$

if the specification language $K$ is conditionally controllable with respect to $\left(G_{1}, G_{2}, G_{k}\right)$ and $\left(E_{1, u}, E_{2, u}, E_{k, u}\right)$.

At the time of submission of this paper, there is no result yet on the necessity condition of the safety. 
The interest in Theorem 7.3 is in the computational saving of the computation of the supervisor, the distributed way of constructing successively the supervisors $S_{k}, S_{1}$, and $S_{2}$ is much less complex than that of the global supervisor constructed for the system $G_{1}\left\|G_{2}\right\| G_{k}$.

\section{CONCLUDING REMARKS}

The concepts of conditional independence of a tuple of generators given a third generator and that of a coordinator for modular discrete-event system have been introduced. It was established that using a coordinator for safety and for nonblockingness in a modular system the composite global supervisor satisfies the safety and the nonblockingness conditions. A construction of the overall supervisor for safety was proposed. More work on coordination control is needed: construction of minimal coordinators should be investigated.

\section{REFERENCES}

[1] C. Cassandras and S. Lafortune, Introduction to discrete event systems. Boston: Kluwer Academic Publishers, 1999.

[2] B. Gaudin and H. Marchand, "Supervisory control of concurrent discrete event systems," IRISA, Rennes, Report Publication interne No. 1593, 2004.

[3] L. Feng, "Computationally efficient supervisory control design for discrete-event systems," Ph.D. dissertation, University of Toronto, Toronto, 2007.

[4] L. Feng and W. Wonham, "Computationally efficient supervisor design: Abstraction and modularity," in Proc. 8th International Workshop on Discrete Event Systems, S. Lafortune and F. Lin, Eds., IEEE. New York: IEEE, 2006, pp. 3-8.

[5] J. Komenda and J. H. van Schuppen, "Supremal sublanguages of general specification languages arising in modular control of discreteevent systems," in Proc. 44th IEEE Conference on Decision and Control. New York: IEEE Press, 2005, pp. 2775-2780.

[6] J. Komenda, J. H. van Schuppen, B. Gaudin, and H. Marchand, "Modular supervisory control with general indecomposable specification languages," in Proc. 44th IEEE Conference on Decision and Control. New York: IEEE Press, 2005, pp. 3474-3479.

[7] J. Komenda and J. H. van Schuppen, "Conditions structurelles dans le contrôle modulaire des systèmes à événements discrets concurrents," in Proceedings Modélisation des Systèmes Ractifs (MSR) 2007, E. Niel and J.-M. Miller, Eds., Ecole Normale Superior de Lyon. Paris: Hermès (Lavoisier), 2007, pp. 53-70.

[8] J. Komenda and J. H. van Schuppen, "Control of discrete-event systems with modular or distributed structure," Theoretical Computer Science, vol. 388, pp. 199-226, 2007.

[9] C. van Putten and J. van Schuppen, "Invariance properties of the conditional independence relation," Ann. Probab., vol. 13, pp. 934945, 1985.

[10] P. Ramadge and W. Wonham, "Supervisory control of a class of discrete event processes," SIAM J. Control Optim., vol. 25, pp. 206230, 1987.

[11] K.Schmidt, B. Gaudin, and H. Marchand, "Modular and decentralized supervisory control of concurrent discrete event systems using reduced system models," in Proc. Int. Workshop on Discrete-Event Systems (WODES.2006). New York: IEEE, 2006, pp. 149-154.

[12] K. Schmidt, "Hierarchical control of decentralized discrete event systems," Ph.D. dissertation, Universiät Erlangen-Nürnberg, Erlangen, 2005.

[13] K. Wong, "On the complexity of projections of discrete-event systems," in Proceedings International Workshop on Discrete-Event Systems (WODES'98). London: IEE, 1998, pp. 201-206.

[14] K. C. Wong and W. M. Wonham, "Modular control and coordination of discrete-event systems," Discrete Event Dynamics Systems, vol. 8, pp. 247-297, 1998.

[15] W. Wonham, Lecture notes on control of discrete-event systems. Toronto: University of Toronto, Department ECE, 2005.

\section{APPENDIX}

In this appendix proofs of Theorem 4.2 and Lemma 4.4 are stated.
Proof: of Theorem $4.2(a)$ is a property known from concurrency theory. Let $L_{1}, L_{2}$ be conditionally independent given $L_{k}$, i.e. $E_{r}\left(L_{1} \| L_{2}\right) \cap E_{1} \cap E_{2} \subseteq E_{k}$. It is easy to show that the triple $\left(L_{1}, L_{2}, L_{c}\right)$ is conditionally shuffle closed. We sketch the proof for $k=1$. Let $t=s_{1} t_{c 1} \in L_{1}\left\|L_{2}\right\| L_{k}$ with $s_{1} \in\left[\left(E_{1 \backslash c}\right) \cup\left(E_{2 \backslash c}\right]^{*}\right.$ and $t_{c 1} \in E_{k}^{*}$. Then $P_{1}(t)=$ $P_{1}\left(s_{1}\right) P_{1}\left(t_{c 1}\right)=P_{1 \backslash c}(t) P_{1}\left(t_{c 1}\right) \in L_{1}, P_{1}(t)=P_{1}\left(s_{1}\right) P_{1}\left(t_{c 1}\right)=$ $P_{1 \backslash c}(t) P_{1}\left(t_{c 1}\right) \in L_{1}$, and $P_{k}(t)=t_{c 1} \in L_{k}$. For any element $\bar{t} \in$ shuffle $\left(P_{1 \backslash c}\left(s_{1}\right), P_{2 \backslash c}\left(s_{1}\right)\right) t_{c 1}=P_{1 \backslash c}^{-1} P_{1 \backslash c}\left(s_{1}\right) \cap P_{2 \backslash c}^{-1} P_{2 \backslash c}\left(s_{1}\right) t_{c 1}$ we get $P_{1}(\bar{t})=P_{1 \backslash c}\left(s_{1}\right) P_{1}\left(t_{c 1}\right) \in L_{1}$. Similarly, $P_{2}(\bar{t}) \in L_{2}$, and finally $\left.P_{k}(\bar{t})=t_{(} c 1\right) \in L_{k}$, hence $\bar{t} \in L_{1}\left\|L_{2}\right\| L_{k}$. The argument can be extended by induction along the string $t \in L_{1}\left\|L_{2}\right\| L_{k}$. (b) $(b 1) \Rightarrow(b 2)$ Let the triple $\left(L_{1}, L_{2}, L_{k}\right)$ is conditionally shuffle closed and let $t, \bar{t} \in E^{*}$ be such that $P_{k}(\bar{t})=$ $P_{k}(t), P_{1 \cup k}(\bar{t})=P_{1 \cup k}(t), P_{2 \cup k}(\bar{t})=P_{2 \cup k}(t)$, and $\bar{t} \in L_{1}\left\|L_{2}\right\| L_{k}$. Let $\bar{t}$ have the following decomposition:

$$
\bar{t}=s_{1} t_{c_{1}} s_{2} \ldots s_{k} t_{c, k} s_{k+1}
$$

with $t_{c_{i}} \in E_{k}^{*}, i=1, \ldots, k$ and $s_{i} \in\left[\left(E_{1} \backslash E_{k}\right) \cup\right.$ $\left.\left(E_{2} \backslash E_{k}\right)\right]^{*}$. Then $P_{k}(t)=P_{k}(\bar{t})=t_{c_{1}} \ldots t_{c, k}$. Also, $P_{1 \cup k}(t)=P_{1 \cup k}(\bar{t})=P_{1 \backslash c}\left(s_{1}\right) t_{c_{1}} \ldots t_{c, k} P_{1 \backslash c}\left(s_{k+1}\right) \quad$ and $P_{2 \cup k}(t)=P_{2 \cup k}(\bar{t})=P_{2 \backslash c}\left(s_{1}\right) t_{c_{1}} \ldots t_{c, k} P_{2 \backslash c}\left(s_{k+1}\right)$. It follows from the assumptions that $t \in$ $\operatorname{shuffle}\left(P_{1 \backslash c}\left(s_{1}\right), P_{2 \backslash c}\left(s_{1}\right)\right) t_{c_{1}} \ldots t_{c, k}$ shuffle $\left(P_{1 \backslash c}\left(s_{k+1}\right) P_{2 \backslash c} s_{k+1}\right)$, i.e. $t \in L_{1}\left\|L_{2}\right\| L_{k}$ using conditional independence.

(b2) $\Leftrightarrow$ (b3) is easy: (b2) can be directly rewritten as $\quad P_{1 \cup k}\left(L_{1}\left\|L_{2}\right\| L_{k}\right)\left\|P_{2 \cup k}\left(L_{1}\left\|L_{2}\right\| L_{k}\right)\right\| P_{k}\left(L_{1}\left\|L_{2}\right\| L_{k}\right) \subseteq$ $L_{1}\left\|L_{2}\right\| L_{k}$, which is the nontrivial inclusion of the definition of conditional decomposability (b3).

$(b 3) \Rightarrow(b 1)$ Let $t, \bar{t} \in E^{*}$ be such that

$$
\bar{t}=s_{1} t_{c_{1}} s_{2} \ldots s_{k} t_{c, k} s_{k+1} \in L_{1}\left\|L_{2}\right\| L_{k}
$$

with $t_{c_{i}} \in E_{k}^{*}, \quad i=1, \ldots, k$ and $s_{i} \in\left[\left(E_{1} \backslash\right.\right.$ $\left.\left.E_{k}\right) \cup\left(E_{2} \backslash E_{k}\right)\right]^{*} \quad i=1, \ldots, k+1$, and let $t \in$ $\operatorname{shuffle}\left(P_{1 \backslash c}\left(s_{1}\right), P_{2 \backslash c}\left(s_{1}\right)\right) t_{c_{1}} \ldots t_{c, k} \operatorname{shuffle}\left(P_{1 \backslash c}\left(s_{k+1}\right) P_{2 \backslash c} s_{k+1}\right)$. Then $P_{k}(\bar{t})=P_{k}(t)=t_{c_{1}} \ldots t_{c, k}, \quad P_{1 \cup k}(\bar{t})=$ $P_{1 \cup k}(t)$, and $\quad P_{2 \cup k}(\bar{t})=P_{2 \cup k}(t), \quad$ and $\bar{t} \in$ $L_{1}\left\|L_{2}\right\| L_{k}$. Then $t \in\left(P_{1 \cup k}\right)^{-1} P_{1 \cup k}\left(L_{1}\left\|L_{2}\right\| L_{k}\right) \cap$ $\left(P_{2 \cup k}\right)^{-1} P_{2 \cup k}\left(L_{1}\left\|L_{2}\right\| L_{k}\right) \cap\left(P_{k}\right)^{-1} P_{k}\left(L_{1}\left\|L_{2}\right\| L_{k}\right)=$ $P_{1 \cup k}\left(L_{1}\left\|L_{2}\right\| L_{k}\right)\left\|P_{2 \cup k}\left(L_{1}\left\|L_{2}\right\| L_{k}\right)\right\| P_{k}\left(L_{1}\left\|L_{2}\right\| L_{k}\right)$ $L_{1}\left\|L_{2}\right\| L_{k}$, hence $t \in L_{1}\left\|L_{2}\right\| L_{k}$ and (b1) holds.

Proof: of Lemma 4.4 (i) It follows easily from Lemma 4.3 with $E_{1}:=E_{k}$ and $E_{2}:=E_{1} \cup E_{2}$ : $P_{k}\left(L_{1}\left\|L_{2}\right\| L_{k}\right)=P_{k}\left(L_{1} \| L_{2}\right) \| P_{k}\left(L_{k}\right)=P_{k}\left(L_{1} \| L_{2}\right) \cap L_{k} \subseteq L_{k}$, because both $P_{k}\left(L_{k}\right)=L_{k}$ and $P_{k}\left(L_{1} \| L_{2}\right)$ are languages over whole $E_{k}$.

(ii) Lemma 4.3 yields $P_{1 \cup k}\left(L_{1}\left\|L_{2}\right\| L_{k}\right)=P_{1 \cup k}\left(\left(L_{1} \| L_{k}\right) \| L_{2}\right)=$ $L_{1}\left\|L_{k}\right\| P_{2 \cap[1 \cup k]}^{2}\left(L_{2}\right)=L_{1} \| L_{k} \cap\left(P_{2 \cap[1 \cup k}^{1 \cup k}\right)^{-1} P_{2 \cap[1 \cup k]}^{2}\left(L_{2}\right) \subseteq$ $L_{1} \| L_{k}$, where $P_{2 \cap[1 \cup k]}^{2}: E_{2}^{*} \rightarrow\left(E_{2} \cap E_{1 \cup k}\right)^{*}$ and $\left(P_{2 \cap[1 \cup k]}^{1 \cup k}\right)^{-1}: \operatorname{Pwr}\left(E_{2} \cap E_{1 \cup k}\right)^{*} \rightarrow \operatorname{Pwr} E_{1 \cup k}^{*}$.

(iii) proof is similar to (ii)

\section{Acknowledgment}

Partial financial support of Grant GA AV No. B100190609 and the Academy of Sciences of the Czech Republic, Institutional Research Plan No. AV0Z10190503. is gratefully acknowledged. 